\title{
Effects of Coriolis force and different basic temperature gradients on Marangoni ferroconvection
}

\author{
I. S. Shivakumara and C. E. Nanjundappa, Bangalore, India \\ Received June 24, 2005 \\ Published online: March 6, 2006 @ Springer-Verlag 2006
}

\begin{abstract}
Summary. The effect of Coriolis force and different forms of basic temperature gradients on the onset of Marangoni ferroconvection in a horizontal layer of ferrofluid is investigated theoretically. The lower boundary is assumed to be rigid-isothermal, while the upper free boundary on which the surface tension acts is non-deformable and insulating to temperature perturbations. The Galerkin technique is used to obtain the critical stability parameters. It is shown that convection sets in as oscillatory motions provided that the Prandtl number is less than unity. A mechanism for suppressing or augmenting Marangoni ferroconvection by rotation, nonlinearity of magnetization and different forms of basic temperature gradients is discussed in detail. It is found that the inverted parabolic temperature profile indicates a reinforcement of stability, whereas the step function temperature profile indicates a diminution of stability. Comparisons of results between the present and the existing ones are made under the limiting conditions and good agreement is found.
\end{abstract}

\section{Introduction}

Convection in ferromagnetic fluids in the presence of a uniform magnetic field, called ferroconvection, is analogous to the Rayleigh-Benard convection in a horizontal layer of an ordinary viscous fluid heated from below and cooled from above. Ferroconvection in a horizontal layer of ferrofluid has been studied extensively because of its diverse applications, namely, energy conversion systems, liquid cooled loudspeakers, magnetic fluid seals and in many other engineering and technological applications [1]-[3]. Finlayson [4] was the first to study the linear stability of ferroconvection in a horizontal layer of ferrofluid heated from below in the presence of a uniform vertical magnetic field. Lalas and Carmi [5] have analyzed the same problem using the energy method. A similar analysis but with the fluid confined between ferromagnetic plates has been carried out by Gotoh and Yamada [6] using the linear stability analysis. Schwab et al. [7] have conducted experiments and their results are found to be in good agreement with [4]. Stiles and Kagan [8] have extended the problem to allow for the dependence of effective shear viscosity on temperature and colloid concentration. Recently, Kaloni and Lou [9] have studied convective instability in a horizontal layer of a magnetic fluid by considering the relaxation time and the rotational viscosity effects. 
The effect of Coriolis force on ferroconvection has also been investigated because the ferrofluids are known to exhibit peculiar characteristics when set to rotation. Das Gupta and Gupta [10] have studied the convective instability in a rotating layer of ferrofluids between two free boundaries. Rudraiah and Sekhar [11] have analyzed the effect of uniform distribution of heat source on the onset of stationary ferroconvection. Venkatasubramanian and Kaloni [12] have discussed the effect of rotation on thermo-convective instability of a horizontal layer of ferrofluid confined between stress-free, rigid-paramagnetic and rigid-ferromagnetic boundaries for uniform temperature gradient. The corresponding problem for ferroconvection in a rotating porous medium is discussed by Sekar et al. [13], and Vaidyanathan et al. [14]. In the latter paper, the effect of magnetic field dependent viscosity is also taken into consideration. The weakly nonlinear instability of a rotating ferromagnetic fluid layer heated from below is discussed by Kaloni and Lou [15].

It is known that, apart from buoyancy, convective instability can also occur due to the local variation of surface tension when the fluid surface is free [16]. This type of convective instability is referred to as Marangoni convection. Much of the available literature on Marangoni convection has been concerned with viscous liquid layers and very little is known about Marangoni convection in ferrofluids, called Marangoni ferroconvection. In view of the fact that heat transfer is greatly enhanced due to convection, Marangoni ferroconvection offers new possibilities for applications in microgravity environments. Qin and Kaloni [17] have studied linear and nonlinear stability problems of combined buoyancy-surface tension effects in a ferrofluid layer heated from below. Recently, Shivakumara et al. [18] have discussed in detail the effect of different forms of basic temperature gradients on the onset of ferroconvection driven by combined surface tension and buoyancy forces.

In view of the fact that rotation gives rise to interesting practical situations, the object of this paper is to study the combined effect of rotation and different forms of basic temperature gradients on the linear stability of Marangoni ferroconvection. In this study the lower rigid boundary is considered to be isothermal and the upper non-deformable free boundary is insulating to temperature perturbations. The resulting eigenvalue problem is solved numerically by employing the Galerkin technique. A comparative study is conducted to analyze the relative effects of different temperature profiles on the onset of convection and with the other works as well under the limiting conditions.

\section{Formulation of the problem}

We consider an infinite horizontal layer of an electrically non-conducting Boussinesq ferromagnetic fluid of depth $d$ permeated by a uniform magnetic field acting normal to the boundaries. The layer is rotating uniformly about its vertical axis with angular velocity $\vec{\Omega}=\Omega \hat{k}$, which is bounded below by a rigid-isothermal surface and above by a non-deformable freeinsulating surface. A temperature drop $\Delta T$ is acting across the boundaries and a Cartesian coordinate system $(x, y, z)$ is used with the origin at the bottom of the surface and the $z$-axis vertically upwards. The surface tension $\sigma$ is assumed to vary linearly with temperature as $\sigma=\sigma_{0}-\sigma_{T} \Delta T$, where $\sigma_{0}$ is the unperturbed value and $\sigma_{T}$ is the rate of change of surface tension with temperature.

The relevant governing equations in the rotating frame of reference are [1], [4], [10]:

$\nabla \cdot \vec{q}=0$

$\rho_{0}\left[\frac{\partial \vec{q}}{\partial t}+(\vec{q} \cdot \nabla) \vec{q}\right]=-\nabla p+\mu_{0}(\vec{M} \cdot \nabla) \vec{H}+\mu \nabla^{2} \vec{q}+2 \rho_{0} \vec{q} \times \vec{\Omega}+\frac{\rho_{0}}{2} \nabla\left(|\vec{\Omega} \times \vec{r}|^{2}\right)$, 
$\left[\rho_{0} C_{V, H}-\mu_{0} \vec{H} \cdot\left(\frac{\partial \vec{M}}{\partial T}\right)_{V, H}\right] \frac{D T}{D t}+\mu_{0} T\left(\frac{\partial \vec{M}}{\partial T}\right)_{V, H} \cdot \frac{D \vec{H}}{D t}=k \nabla^{2} T$

$\nabla \cdot \vec{B}=0, \quad \nabla \times \vec{H}=0$,

$\vec{B}=\mu_{0}(\vec{M}+\vec{H})$

$\vec{M}=\frac{M}{H}(H, T) \vec{H}$,

$M=M_{0}+\chi\left(H-H_{0}\right)-K(T-\bar{T})$,

where $\vec{q}=(u, v, w)$ is the velocity, $p$ is the pressure, $T$ is the temperature, $\vec{H}$ is the magnetic field, $\vec{M}$ is the magnetization, $C_{V, H}$ is the specific heat at constant volume and magnetic field, $\vec{B}$ is the magnetic induction, $\mu$ is the coefficient of viscosity, $\mu_{0}$ is the magnetic permeability of vacuum, $\rho_{0}$ is the reference density, $k$ is the thermal conductivity, $\bar{T}$ is the average temperature, $\chi=(\partial M / \partial H)_{H_{0}, \bar{T}}$ is the magnetic susceptibility, $K=-(\partial M / \partial T)_{H_{0}, \bar{T}}$ is the pyromagnetic coefficient, $M_{0}=M\left(H_{0}, \bar{T}\right)$, and $H_{0}$ is the imposed uniform vertical magnetic field.

The basic state is given by

$\vec{q}=0, \quad p=p_{b}(z), \quad-\frac{d T_{b}}{d z}=f(z)$,

$\vec{H}_{b}=\left[H_{0}+\frac{K\left(T_{b}-\bar{T}\right)}{1+\chi}\right] \hat{k}, \quad \vec{M}_{b}=\left[M_{0}-\frac{K\left(T_{b}-\bar{T}\right)}{1+\chi}\right] \hat{k}$,

where $\hat{k}=(0,0,1)$ is the unit vector in the $z$-direction, the subscript $b$ denotes the basic state and $f(z)$ is the basic temperature gradient, such that $\int_{0}^{d} f(z) d z=-\frac{\Delta T}{d}$.

We shall analyze the stability of the basic state by introducing the following perturbations:

$\vec{q}=\vec{q}^{\prime}, \quad p=p_{b}(z)+p^{\prime}, \quad T=T_{b}(z)+T^{\prime}, \quad \vec{H}=\vec{H}_{b}(z)+\vec{H}^{\prime}, \quad \vec{M}=\vec{M}_{b}(z)+\vec{M}^{\prime}$,

where the primed quantities are perturbed ones and they are assumed to be small.

Substituting Eq. (9) into Eqs. (5) and (6) and using Eqs. (4) and (7), we get

$H_{i}^{\prime}+M_{i}^{\prime}=\left(\frac{1+M_{0}}{H_{0}}\right) H_{i}^{\prime}, \quad i=1,2$,

$H_{3}^{\prime}+M_{3}^{\prime}=(1+\chi) H_{3}^{\prime}-K T^{\prime}$,

where we have assumed $K\left(T_{b}-\bar{T}\right) \ll(1+\chi) H_{0}$.

Using Eq. (9) in Eq. (2), linearizing and then taking curl on the resulting equation (after neglecting primes), the $z$-component can be written as

$\rho_{0} \frac{\partial \xi}{\partial t}=\mu \nabla^{2} \xi+2 \rho_{0} \Omega \frac{\partial w}{\partial z}$,

which is the vorticity transport equation and $\xi=\partial v / \partial x-\partial u / \partial y$ is the $z$-component of vorticity. Substituting Eq. (9) in Eq. (2), linearizing, taking curl twice and then using Eqs. (10) and (11) together with $\vec{H}^{\prime}=\nabla \varphi^{\prime}$, the $z$-component of the resulting equation can be written as (after neglecting the primes)

$\left(\rho_{0} \frac{\partial}{\partial t}-\mu \nabla^{2}\right) \nabla^{2} w=-2 \rho_{0} \Omega \frac{\partial \xi}{\partial z}-\mu_{0} K f(z) \frac{\partial}{\partial z}\left(\nabla_{1}^{2} \varphi\right)+\frac{\mu_{0} K^{2} f(z)}{1+\chi} \nabla_{1}^{2} T$. 
As before, using Eq. (9) in Eq. (3), and after linearizing, the equation obtained is (neglecting primes)

$\rho_{0} C_{0} \frac{\partial T}{\partial t}-k \nabla^{2} T-\mu_{0} K T_{0} \frac{\partial}{\partial t}\left(\frac{\partial \varphi}{\partial z}\right)=\left(\rho_{0} C_{0}-\frac{\mu_{0} K^{2} T_{0}}{(1+\chi)}\right) w f(z)$,

where $\rho_{0} C_{0}=\rho_{0} C_{V, H}+\mu_{0} K H_{0}, \nabla_{1}^{2}=\partial^{2} / \partial x^{2}+\partial^{2} / \partial y^{2}$ and $\nabla^{2}=\nabla_{1}^{2}+\partial^{2} / \partial z^{2}$.

Finally Eqs. (4.1, 2), after using Eqs. (10) and (11) together with $\vec{H}^{\prime}=\nabla \varphi^{\prime}$ become (neglecting primes)

$$
\left(1+\frac{M_{0}}{H_{0}}\right) \nabla_{1}^{2} \varphi+(1+\chi) \frac{\partial^{2} \varphi}{\partial z^{2}}-K \frac{\partial T}{\partial z}=0 .
$$

We perform normal mode expansion of the dependent variables in the form

$\{w, T, \varphi, \xi\}=\{W(z), \theta(z), \varphi(z), \xi(z)\} \exp [i(l x+m y)+\omega t]$,

where $l$ and $m$ are wave numbers in the $x$ - and $y$-direction, respectively, $\omega$ is the growth rate, $W(z), \theta(z), \varphi(z)$ and $\xi(z)$ are the amplitudes of the $z$-component of the perturbation velocity, perturbation temperature, perturbation magnetization and the $z$-component of the perturbation vorticity, respectively.

Substituting Eq. (16) into Eqs. (12)-(15), we get

$$
\begin{aligned}
& {\left[\rho_{0} \omega-\mu\left(D^{2}-a^{2}\right)\right]\left(D^{2}-a^{2}\right) W=a^{2} \mu_{0} K f(z) D \varphi-\frac{a^{2} \mu_{0} K^{2} f(z)}{1+\chi} \theta-2 \rho_{0} \Omega D \xi,} \\
& \omega \theta-\kappa\left(D^{2}-a^{2}\right) \theta-\frac{\mu_{0} K T_{0}}{\rho_{0} C_{0}} \omega D \varphi=\left(1-\frac{\mu_{0} K^{2} T_{0}}{(1+\chi) \rho_{0} C_{0}}\right) W f(z), \\
& (1+\chi) D^{2} \varphi-\left(\frac{1+M_{0}}{H_{0}}\right) a^{2} \varphi-K D \theta=0, \\
& \rho_{0} \omega \xi=\mu\left(D^{2}-a^{2}\right) \xi+2 \rho_{0} \Omega D W,
\end{aligned}
$$

where $D=d / d z$ is the differential operator and $a=\sqrt{l^{2}+m^{2}}$ is the overall horizontal wavenumber.

Equations (17)-(20) are nondimensionalized by setting

$$
\begin{aligned}
W^{*} & =\frac{d}{v} W, \quad a^{*}=a \mathrm{~d}, \quad \mathrm{D}^{*}=\mathrm{dD}, \quad \mathrm{t}^{*}=\frac{v}{d^{2}} t, \quad \xi^{*}=\frac{d^{2}}{v} \xi, \\
\omega^{*} & =\frac{d^{2}}{v} \omega, \quad \theta^{*}=\frac{\kappa}{\beta v d} \theta, \quad \varphi^{*}=\frac{(1+\chi) \kappa}{K \beta v d^{2}} \varphi, \quad f(z)^{*}=\frac{1}{\beta} f(z)\left(\beta=-\frac{\Delta \mathrm{T}}{d}\right) .
\end{aligned}
$$

Thus Eqs. (17)-(20) become (after neglecting the asterisks)

$$
\begin{aligned}
& \left(D^{2}-a^{2}-\omega\right)\left(D^{2}-a^{2}\right) W=\mathrm{Ta}^{1 / 2} D \xi+N a^{2} f(z) \theta-N a^{2} f(z) D \varphi, \\
& \left(D^{2}-a^{2}-\omega \operatorname{Pr}\right) \theta+\omega \operatorname{Pr} M_{2} D \varphi=-\left(1-M_{2}\right) W f(z), \\
& \left(D^{2}-a^{2} M_{3}\right) \varphi-D \theta=0, \\
& \left(D^{2}-a^{2}-\omega\right) \xi=-\mathrm{Ta}^{1 / 2} D W .
\end{aligned}
$$

Here $\mathrm{Ta}^{1 / 2}=2 \Omega d^{2} / v$ is the square root of the Taylor number, $N=\mu_{0} K^{2} \beta^{2} d^{4} /(1+\chi) \kappa \mu$ is the magnetic Rayleigh number, $M_{3}=\left(1+M_{0} / H_{0}\right) /(1+\chi)$ is the measure of nonlinearity of magnetisation, $\operatorname{Pr}=v / \kappa$ is the Prandtl number, and $M_{2}=\mu_{0} T_{0} K^{2} / \rho_{0} C_{0}(1+\chi)$ is the nondi- 
mensional parameter and is neglected in the subsequent analysis since its value is negligible [4], and $f(z)$ is the nondimensional basic temperature gradient, such that $\int_{0}^{1} f(z) d z=1$.

The corresponding boundary conditions for the perturbed nondimensional variables take the form

$W=D W=\varphi=\theta=\xi=0 \quad$ at $z=0$,

$W=D^{2} W+\operatorname{Ma} a^{2} \theta=D \varphi=D \theta=D \xi=0 \quad$ at $z=1$,

where $\mathrm{Ma}=\sigma_{T} \Delta T d / \mu \kappa$ is the Marangoni number.

To investigate the effect of nonuniform temperature gradients on the convective instability, the following types of basic temperature profiles are considered:

Model 1: Linear temperature profile:

$f(z)=1$.

Model 2: Piecewise linear temperature profile heating from below:

$f(z)=\left\{\begin{array}{cl}\frac{1}{\varepsilon} & \text { for } 0 \leq z<\varepsilon \\ 0 & \text { for } \varepsilon<z \leq 1 .\end{array}\right.$

Model 3: Piecewise linear temperature profile cooling from above:

$f(z)=\left\{\begin{array}{cc}0 & \text { for } 0 \leq z<\varepsilon \\ \frac{1}{(1-\varepsilon)} & \text { for } \varepsilon<z \leq 1 .\end{array}\right.$

Model 4: Step function temperature profile:

$f(z)=\delta(z-\varepsilon)$.

Model 5: Inverted parabolic temperature profile:

$f(z)=2(1-z)$.

Model 6: Parabolic temperature profile:

$f(z)=2 z$.

Regarding the applicability of the above temperature profiles in the situation considered, it may be noted that these can be achieved by sudden heating or cooling of boundaries and also by a uniform volumetric heat source (for details see [19]).

\section{Method of solution}

The governing equations (22)-(25) together with the boundary conditions (26) and (27) constitute an eigenvalue problem with $\mathrm{Ma}$ as an eigenvalue. To solve the resulting eigenvalue problem, the Galerkin technique is used. Accordingly, the variables are written in a series of basis functions as

$W=\sum_{i=1}^{n} A_{i} W_{i}(z), \quad \theta(z)=\sum_{i=1}^{n} B_{i} \theta_{i}(z), \quad \varphi(z)=\sum_{i=1}^{n} C_{i} \varphi_{i}(z), \quad \xi=\sum_{i=1}^{n} E_{i} \xi_{i}(z)$, 
where the trial functions $W_{i}(z), \theta_{i}(z), \varphi_{i}(z)$ and $\xi_{i}(z)$ will be generally chosen in such a way that they satisfy the respective boundary conditions, and $A_{i}, B_{i}, C_{i}$, and $E_{i}$ are constants.

Substituting Eq. (28) into Eqs. (22)-(25), multiplying the resulting momentum equation by $W_{j}(z)$, the energy equation by $\theta_{j}(z)$, the magnetic potential equation by $\varphi_{j}(z)$ and the vorticity equation by $\xi_{j}(z)$, performing the integration by parts with respect to $z$ between $z=0$ and $z=1$ and using the boundary conditions (26) and (27), we obtain the following system of linear homogeneous algebraic equations:

$C_{j i} A_{i}+D_{j i} B_{i}+E_{j i} C_{i}+F_{j i} E_{i}=0$,

$G_{j i} A_{i}+H_{j i} B_{i}=0$,

$I_{j i} B_{i}+J_{j i} C_{i}=0$,

$K_{j i} A_{i}+L_{j i} E_{i}=0$.

The coefficients $C_{j i}-L_{j i}$ involve the inner products of the basis functions and are given by

$C_{j i}=\left\langle D^{2} W_{j} D^{2} W_{i}\right\rangle+\left(2 a^{2}+\omega\right)\left\langle D W_{j} D W_{i}\right\rangle+a^{2}\left(a^{2}+\omega\right)\left\langle W_{j} W_{i}\right\rangle$,

$D_{j i}=-a^{2} N\left\langle f(z) \theta_{j} W_{i}\right\rangle+a^{2} \mathrm{Ma} D W_{j}(1) \theta_{i}(1)$,

$E_{j i}=a^{2} N\left\langle f(z) W_{j} D \varphi_{i}\right\rangle$,

$F_{j i}=-T a^{1 / 2}\left\langle W_{j} D \zeta_{i}\right\rangle$,

$G_{j i}=-\left\langle f(z) \theta_{j} W_{i}\right\rangle$,

$H_{j i}=\left\langle D \theta_{j} D \theta_{i}\right\rangle+\left(a^{2}+\omega \operatorname{Pr}\right)\left\langle\theta_{j} \theta_{i}\right\rangle$,

$I_{j i}=\left\langle\varphi_{j} D \theta_{i}\right\rangle$,

$J_{j i}=\left\langle D \varphi_{j} D \varphi_{i}\right\rangle+a^{2} M_{3}\left\langle\varphi_{j} \varphi_{i}\right\rangle$,

$K_{j i}=-\mathrm{Ta}^{1 / 2}\left\langle\zeta_{j} D W_{i}\right\rangle$,

$L_{j i}=\left\langle D \zeta_{j} D \zeta_{i}\right\rangle+\left(a^{2}+\omega\right)\left\langle\zeta_{j} \zeta_{i}\right\rangle$

where the inner product is defined as $\langle\cdots \cdots\rangle=\int_{0}^{1}(\cdots) d z$.

The above set of homogeneous algebraic equations can have a non-trivial solution if and only if

$\left|\begin{array}{cccc}C_{j i} & D_{j i} & E_{j i} & F_{j i} \\ G_{j i} & H_{j i} & 0 & 0 \\ 0 & I_{j i} & J_{j i} & 0 \\ K_{j i} & 0 & 0 & L_{j i}\end{array}\right|=0$.

We select the trial functions as

$W_{i}=z^{i+1}-z^{i+2}, \quad \theta_{i}=z^{i}-\frac{z^{i+1}}{2}, \quad \varphi_{i}=z^{i+1}-\frac{2}{3} z^{i+2} \quad$ and $\quad \zeta_{i}=z^{i+1}-\frac{2}{3} z^{i+2}$,

such that they satisfy all the corresponding boundary conditions except the one, namely $D^{2} W+$ Ma $a^{2} \theta=0$ at $z=1$, but the residual from this equation is included as a residual from the differential equation.

At this juncture, it would be instructive to look at the results for $i=j=1$ and for this order Eq. (33) gives the following characteristic equation: 


$$
\mathrm{Ma}=-\frac{\left(\eta_{1}+2 \omega \mathrm{Pr}\right)}{1575 a^{2}\langle f(z) W \theta\rangle}\left[\frac{147 \mathrm{Ta}}{\left(\eta_{2}+13 \omega\right)}+2\left(\eta_{3}+\eta_{4} \omega\right)\right]-\frac{63 N\langle f(z) W D \varphi\rangle}{2 \eta_{5}}-2 N\langle f(z) W \theta\rangle
$$

where $\eta_{1}=2 a^{2}+5, \eta_{2}=42+13 a^{2}, \eta_{3}=a^{4}+28 a^{2}+420, \eta_{4}=14+a^{2}$ and $\eta_{5}=42+13$ $M_{3} a^{2}$.

To examine the stability of the system, we take $\omega=i \omega$ in Eq. (35) and clear the complex quantities from the denominator of Eq. (35), to get

$$
\begin{aligned}
\mathrm{Ma}= & -\frac{1}{1575 a^{2}\langle f(z) W \theta\rangle}\left[\frac{147 \mathrm{Ta}\left(\eta_{1} \eta_{2}+26 \omega^{2} \mathrm{Pr}\right)}{\left(\eta_{2}^{2}+169 \omega^{2}\right)}+2\left(\eta_{1} \eta_{3}-2 \omega^{2} \eta_{4} \mathrm{Pr}\right)\right] \\
& -2 N\langle f(z) W \theta\rangle-\frac{63 N\langle f(z) W D \varphi\rangle}{2 \eta_{5}}+i \omega \Delta,
\end{aligned}
$$

where

$$
\Delta=-\frac{1}{1575 a^{2}\langle f(z) W \theta\rangle}\left[\frac{147 \mathrm{Ta}\left(2 \eta_{2} \operatorname{Pr}-13 \eta_{1}\right)}{\left(\eta_{2}^{2}+169 \omega^{2}\right)}+2\left(2 \eta_{3} \operatorname{Pr}++\eta_{1} \eta_{4}\right)\right] .
$$

Since Ma is a physical quantity it must be real, so that it implies either $\omega=0$ or $\Delta=0(\omega \neq 0)$, and accordingly the condition for steady and oscillatory onset is obtained.

The steady onset is governed by $\omega=0$ and it occurs at $\mathrm{Ma}=\mathrm{Ma}^{s}$, where

$\mathrm{Ma}^{s}=-\frac{\eta_{1}}{1575 a^{2}\langle f(z) W \theta\rangle}\left(\frac{147 \mathrm{Ta}}{\eta_{2}}+2 \eta_{3}\right)-2 N\langle f(z) W \theta\rangle-\frac{63 N\langle f(z) W D \varphi\rangle}{2 \eta_{5}}$.

The oscillatory convection occurs at $\mathrm{Ma}=\mathrm{Ma}^{0}$, where

$\mathrm{Ma}^{0}=-\frac{2\left(a_{1} a_{4}^{2}+a_{2} a_{4}+a_{3}\right)}{1575 a_{4} a^{2}\langle f(z) W \theta\rangle}-2 N\langle f(z) W \theta\rangle-\frac{63 N\langle f(z) W D \varphi\rangle}{2 \eta_{5}}$.

Here $\quad a_{1}=\eta_{1} \eta_{2}-\frac{26}{169} \operatorname{Pr} \eta_{2}^{2}, \quad a_{2}=\eta_{1} \eta_{3}+\frac{2}{169} \operatorname{Pr} \eta_{4} \eta_{2}^{2}+\frac{147}{13} \mathrm{Ta} \operatorname{Pr}, \quad a_{3}=-\frac{147}{169} \mathrm{Ta} \operatorname{Pr} \eta_{4}$, and $a_{4}=\frac{\eta_{1} \eta_{4}+2 \operatorname{Pr} \eta_{3}}{13 \eta_{1}-26 \operatorname{Pr} \eta_{2}}$.

The corresponding frequency of oscillations is given by

$\omega^{2}=-\frac{\eta_{2}^{2}}{169}+\frac{147 \mathrm{Ta}}{26 \eta_{4}}\left[\frac{1-2 \beta_{1} \operatorname{Pr}}{1+2 \beta_{2} \operatorname{Pr}}\right]$

where

$\beta_{1}=\frac{42+13 a^{2}}{65+26 a^{2}} \quad$ and $\quad \beta_{2}=\frac{a^{4}+28 a^{2}+420}{2 a^{4}+33 a^{2}+70}$.

For the occurrence of oscillatory onset $\omega^{2}$ should be positive and the necessary conditions for the same are

$\operatorname{Pr}<\frac{\left(a^{2}+2.5\right)}{\left(a^{2}+3.23\right)} \quad$ and $\quad \operatorname{Ta}>\frac{26}{24843} \eta_{2}^{2} \eta_{4}\left[\frac{1+2 \beta_{2} \operatorname{Pr}}{1-2 \beta_{1} \operatorname{Pr}}\right]$.

It is thus evident that for the oscillatory onset to exist the Prandtl number Pr should be less than unity as observed in the classical viscous liquids. But for most of the ferrofluids, whether they are water based or any other organic liquid based, the Prandtl number is greater than unity and hence the overstability is not a preferred mode of instability [12], [15].

In what follows we restrict ourselves to the case of steady onset and put $\omega=0$ in Eq. (33). Now this determinantal equation leads to the characteristic equation giving the Marangoni 
number Ma as a function of the wavenumber $a$, basic temperature gradient $f(z)$ and the parameters $\mathrm{N}, \mathrm{M}_{3}$ and Ta. The inner products involved in the determinant were evaluated analytically in order to avoid errors in the numerical integration. The critical Marangoni numbers $\mathrm{Ma}_{c i}(i=1$ to 6$)$ are obtained by minimizing respectively $\mathrm{Ma}_{i}(i=1$ to 6$)$ with respect to the wavenumber $a$ and thermal depth $\varepsilon$ (in the case of Models 2-4). Computations reveal that the convergence in finding $\mathrm{Ma}_{c i}(i=1$ to 6 ) crucially depends on the value of $\mathrm{Ta}$, and for higher value of Ta more terms in the expansion given by Eq. (28) were found to be required. The results presented here are for $i=j=8$, the order at which the convergence is achieved, in general.

\section{Results and discussion}

The linear stability theory is used to investigate the combined effects of Coriolis force and different forms of basic temperature gradients on Marangoni ferroconvection in a rotating ferrofluid layer. The lower boundary is taken to be rigid-isothermal and the upper nondeformable free boundary is insulating to temperature perturbations. The Galerkin technique is used to find the eigenvalues as this technique is found to be more convenient to tackle different basic temperature profiles.

To know the validity of our solution procedure, first the critical values $\left(\mathrm{Ma}_{\mathrm{c}}, \mathrm{a}_{\mathrm{c}}, \varepsilon_{\mathrm{c}}\right)$ obtained from the present study under the limiting conditions are compared with those of Vidal and Acrivos [20] and Lebon and Cloot [19] in Tables 1 and 2, respectively. The results tabulated in Table 1 for different values of Ta are for $f(z)=1$ and $N=0$ (i.e., Marangoni convection for non-ferrofluids), while the results tabulated in Table 2 are for $N=0, \mathrm{Ta}=0$ and for different basic temperature profiles.

From these tables, it is evident that there is an excellent agreement between the present and the previously published results. Further, it may be noted that an increase in the value of Ta is

Table 1. Comparison of $\mathrm{Ma}_{\mathrm{c}}$ and $\mathrm{a}_{\mathrm{c}}$ for different values of $\mathrm{Ta}$ when $N=0$

\begin{tabular}{ccccc}
\hline Ta & \multicolumn{2}{l}{ Vidal and Acrivos [20] } & & \multicolumn{2}{c}{ Present study } \\
\cline { 2 - 3 } \cline { 5 - 5 } & $\mathrm{Ma}_{\mathrm{c}}$ & $\mathrm{a}_{\mathrm{c}}$ & $\mathrm{Ma}_{\mathrm{c}}$ & $\mathrm{a}_{\mathrm{c}}$ \\
\hline 0 & 80 & 2.0 & 79.61 & 1.99 \\
$10^{2}$ & 92 & 2.2 & 91.31 & 2.17 \\
$10^{3}$ & 164 & 3.0 & 163.11 & 2.97 \\
$10^{4}$ & 457 & 5.0 & 456.23 & 4.99 \\
$10^{5}$ & 1400 & 8.6 & 1398.36 & 8.86 \\
\hline
\end{tabular}

Table 2. Comparison of $\mathrm{Ma}_{\mathrm{c}}, \mathrm{a}_{\mathrm{c}}$ and $\varepsilon_{c}$ for different basic temperature profiles when $N=0$ and $\mathrm{Ta}=0$

\begin{tabular}{|c|c|c|c|c|c|c|}
\hline \multirow[t]{2}{*}{ Nature of temperature profile } & \multicolumn{3}{|c|}{ Lebon and Cloot [19] } & \multicolumn{3}{|c|}{ Present study } \\
\hline & $\mathrm{Ma}_{\mathrm{c}}$ & $\mathrm{a}_{\mathrm{c}}$ & $\varepsilon_{\mathrm{c}}$ & $\mathrm{Ma}_{\mathrm{c}}$ & $\mathrm{a}_{\mathrm{c}}$ & $\varepsilon_{\mathrm{c}}$ \\
\hline Linear temperature profile & 79.61 & 1.99 & - & 79.61 & 1.993 & - \\
\hline $\begin{array}{l}\text { Piecewise linear profile heating } \\
\text { from below }\end{array}$ & 78.1 & 2.03 & 0.96 & 78.1 & 1.980 & 0.959 \\
\hline $\begin{array}{l}\text { Piecewise linear profile cooling from } \\
\text { above }\end{array}$ & 42.62 & 2.25 & 0.675 & 42.63 & 2.259 & 0.678 \\
\hline Step function profile & 34.3 & 2.305 & 0.815 & 34.27 & 2.226 & 0.813 \\
\hline
\end{tabular}


Table 3. Values of $\mathrm{Ma}_{\mathrm{c} i}(i=1-6)$ for different values of Ta and $N$ when $M_{3}=1$

\begin{tabular}{|c|c|c|c|c|c|c|c|c|c|c|c|c|c|}
\hline $\mathrm{Ta}$ & $\mathrm{N}$ & $\mathrm{Ma}_{\mathrm{c} 1}$ & $\mathrm{a}_{\mathrm{c}}$ & $\mathrm{Ma}_{\mathrm{c} 2}$ & $a_{c}$ & $\mathrm{Ma}_{\mathrm{c} 3}$ & $a_{c}$ & $\mathrm{Ma}_{\mathrm{c} 4}$ & $a_{c}$ & $\mathrm{Ma}_{\mathrm{c} 5}$ & $\mathrm{a}_{\mathrm{c}}$ & $\mathrm{Ma}_{\mathrm{c} 6}$ & $a_{c}$ \\
\hline \multirow[t]{8}{*}{0} & 0 & 79.61 & 1.993 & 78.16 & 1.978 & 42.63 & 2.259 & 34.27 & 2.226 & 130.91 & 1.844 & 56.91 & 2.075 \\
\hline & 100 & 3.57 & 1.969 & 66.34 & 1.949 & 30.93 & 2.117 & 17.96 & 1.969 & 119.05 & 1.845 & 45.06 & .028 \\
\hline & 183.625 & 59.22 & 1.951 & 56.18 & 1.929 & 20.33 & 2.034 & 0.0 & 1.905 & 108.84 & 1.847 & 35.02 & .939 \\
\hline & 334.900 & 42.06 & 1.924 & 37.13 & 1.901 & 0.0 & 1.934 & & & 90.35 & 1.854 & 16.56 & 1.900 \\
\hline & 468.234 & 26.67 & 1.906 & 19.58 & 1.890 & & & & & 73.82 & 1.865 & 0.0 & 1.939 \\
\hline & 610.840 & 9.95 & 1.892 & 0.0 & 1.898 & & & & & 55.88 & 1.882 & & \\
\hline & 694.631 & 0.0 & 1.888 & & & & & & & 45.19 & 1.895 & & \\
\hline & 1038.800 & & & & & & & & & 0.0 & 1.987 & & \\
\hline \multirow[t]{8}{*}{10} & 0 & 80.85 & 2.012 & 79.40 & 1.998 & 43.09 & 2.284 & 34.66 & 2.251 & 133.45 & .862 & 57.71 & 2.095 \\
\hline & 100 & 69.86 & 1.989 & 67.78 & 1.970 & 31.49 & 2.141 & 18.56 & 1.994 & 121.67 & 63 & 91 & 2.049 \\
\hline & 186.840 & 60.18 & 1.970 & 57.12 & 1.948 & 20.56 & 2.055 & 0.0 & 1.927 & 110.97 & 1.865 & 35.52 & 2.014 \\
\hline & 340.530 & 42.80 & 1.944 & 37.82 & 1.920 & 0.0 & 1.955 & & & 92.16 & 1.873 & 16.84 & 1.960 \\
\hline & 476.595 & 27.13 & 1.926 & 19.94 & 1.911 & & & & & 75.25 & 1.885 & 0.0 & 1.921 \\
\hline & 621.740 & 10.14 & 1.914 & 0.0 & 1.921 & & & & & 56.94 & 1.903 & & \\
\hline & 707.263 & 0.0 & 1.910 & & & & & & & 45.99 & 1.917 & & \\
\hline & 1055.630 & & & & & & & & & 0.0 & 2.015 & & \\
\hline \multirow[t]{8}{*}{$10^{2}$} & 0 & 91.31 & 2.166 & 89.74 & 2.152 & 46.81 & 2.485 & 37.79 & 2.452 & 155.29 & 2.001 & 64.33 & 2.254 \\
\hline & 100 & 80.69 & 2.145 & 78.37 & 2.127 & 35.99 & 2.339 & 23.24 & & 143.24 & 3 & & 2.213 \\
\hline & 213.930 & 68.29 & 2.124 & 65.01 & 2.103 & 22.40 & 2.225 & 0.0 & 2.098 & 129.33 & 2.008 & 39.76 & 2.172 \\
\hline & 387.710 & 49.06 & 2.101 & 43.67 & 2.078 & 0.0 & 2.120 & & & 107.75 & 2.021 & 19.24 & 2.121 \\
\hline & 547.229 & 31.01 & 2.087 & 22.93 & 2.076 & & & & & 87.52 & 2.039 & 0.0 & 2.086 \\
\hline & 713.110 & 11.83 & 2.083 & 0.0 & 2.105 & & & & & 66.00 & 2.676 & & \\
\hline & 813.660 & 0.0 & 2.085 & & & & & & & 52.69 & 2.090 & & \\
\hline & 1193.430 & & & & & & & & & 0.0 & 2.234 & & \\
\hline \multirow[t]{8}{*}{$10^{3}$} & 0 & 163.11 & 2.971 & 160.43 & 2.952 & 66.36 & 3.605 & 54.48 & 3.541 & 329.35 & 2.718 & 107.81 & 3.086 \\
\hline & 100 & 154.23 & 2.956 & 151.13 & 2.936 & 59.04 & 3.461 & 44.59 & 3.365 & 316.94 & 2.721 & 98.48 & 3.059 \\
\hline & 405.813 & 125.98 & 2.924 & 121.10 & 2.896 & 32.34 & 3.135 & 0.0 & 3.080 & 277.49 & 2.741 & 68.93 & 2.996 \\
\hline & 716.400 & 95.47 & 2.912 & 44.03 & 2.944 & 0.0 & 2.995 & & & 234.75 & 2.780 & 37.30 & 2.965 \\
\hline & 1062.670 & 59.07 & 2.932 & 25.59 & 3.093 & & & & & 183.20 & 2.853 & 0.0 & 2.973 \\
\hline & 1334.430 & 28.41 & 2.974 & 0.0 & 3.132 & & & & & 138.95 & 2.941 & & \\
\hline & 1570.140 & 0.00 & 3.033 & & & & & & & 96.88 & 3.047 & & \\
\hline & 2025.880 & & & & & & & & & 0.0 & 3.352 & & \\
\hline
\end{tabular}

to increase $\mathrm{Ma}_{\mathrm{c}}$ and $\mathrm{a}_{\mathrm{c}}$, and thus having a stabilizing effect on the system (see Table 1). Also, in the notation of the present study, it is noted that $\mathrm{Ma}_{\mathrm{c} 4}<\mathrm{Ma}_{\mathrm{c} 3}<\mathrm{Ma}_{\mathrm{c} 2}<\mathrm{Ma}_{\mathrm{c} 4}$ indicating that the non-uniform basic temperature gradients promote instability (see Table 2).

The results obtained for the complete problem for different basic temperature profiles (i.e., Models 1-6) are tabulated in Tables 3 and 4 for $\mathrm{M}_{3}=1$ and 2, respectively, for different values of Ta and $N$. A glance at the tabulated values reveals that the magnetic Rayleigh number has a destabilizing effect on the system. In fact there is a strong coupling between the magnetic Rayleigh and the Marangoni numbers. That is, when the buoyancy is predominant the surface tension effect becomes negligible and the extent to which the surface tension effect is diminished due to $N$ depends on the form of basic temperature gradient $f(z)$, the nonlinearity of magnetization as well as on the strength of rotation. It can be seen that the surface tension effect diminishes for $\mathrm{Ta}=0,10$ and $10^{2}$ at a lesser value of $N$ for $M_{3}=1$, while for $\mathrm{Ta} \geq 10^{3}$ a similar effect is noticed for $M_{3}=2$. Nonetheless, the critical Marangoni number increases with an increase in the Taylor number and this indicates the presence of Coriolis force due to rotation is to reduce the intensity of Marangoni ferroconvection. A comparison of critical Marangoni numbers among different forms of basic temperature profiles shows that 
Table 4. Values of $\mathrm{Ma}_{\mathrm{c} i}(i=1-6)$ for different values of Ta and $N$ when $M_{3}=2$

\begin{tabular}{|c|c|c|c|c|c|c|c|c|c|c|c|c|c|}
\hline $\mathrm{Ta}$ & $N$ & $\mathrm{Ma}_{\mathrm{c} 1}$ & $\mathrm{a}_{\mathrm{c}}$ & $\mathrm{Ma}_{\mathrm{c} 2}$ & $a_{c}$ & $\mathrm{Ma}_{\mathrm{c} 3}$ & $\mathrm{a}_{\mathrm{c}}$ & $\mathrm{Ma}_{\mathrm{c} 4}$ & $a_{c}$ & $\mathrm{Ma}_{\mathrm{c} 5}$ & $a_{c}$ & $\mathrm{Ma}_{\mathrm{c} 6}$ & $\mathrm{a}_{\mathrm{c}}$ \\
\hline \multirow[t]{8}{*}{0} & 0 & 79.61 & 1.993 & 78.16 & 1.978 & 42.63 & 2.259 & 34.27 & 2.226 & 130.91 & 1.844 & 56.91 & 2.075 \\
\hline & 100 & 68.98 & 1.979 & 66.77 & 1.959 & 31.21 & 2.137 & 18.60 & 2.069 & 119.32 & 1.851 & 45.44 & 2.044 \\
\hline & 194.744 & 58.76 & 1.969 & 55.64 & 1.947 & 19.47 & 2.065 & 0.0 & 2.071 & 108.19 & 1.860 & 34.41 & 2.019 \\
\hline & 342.630 & 42.53 & 1.959 & 37.52 & 1.938 & 0.0 & 2.003 & & & 90.54 & 1.879 & 16.89 & 1.989 \\
\hline & 482.545 & 26.85 & 1.957 & 19.42 & 1.952 & & & & & 73.49 & 1.904 & 0.0 & 1.971 \\
\hline & 624.260 & 10.63 & 1.964 & 0.0 & 2.996 & & & & & 55.83 & 1.938 & & \\
\hline & 715.481 & 0.0 & 1.973 & & & & & & & 44.21 & 1.966 & & \\
\hline & 1045.52 & & & & & & & & & 0.0 & 2.126 & & \\
\hline \multirow[t]{8}{*}{10} & 0 & 80.85 & 2.012 & 79.39 & 1.998 & 43.09 & 2.284 & 34.67 & 2.251 & 133.45 & 1.862 & 57.71 & 2.095 \\
\hline & 100 & 70.26 & 1.999 & 68.05 & 1.979 & 31.77 & 2.162 & 19.13 & 2.093 & 121.84 & 1.896 & 46.28 & 2.064 \\
\hline & 197.797 & 59.74 & 1.989 & 56.60 & 1.967 & 19.72 & 2.087 & 0.0 & 2.096 & 110.4 & 1.879 & 34.93 & 2.039 \\
\hline & 348.120 & 43.28 & 1.979 & 38.22 & 1.959 & 0.0 & 2.025 & & & 92.35 & 1.898 & 17.18 & 2.011 \\
\hline & 490.752 & 27.31 & 1.978 & 19.77 & 1.974 & & & & & 74.91 & 1.925 & 0.0 & 1.993 \\
\hline & 634.641 & 10.85 & 1.986 & 0.0 & 2.022 & & & & & 56.89 & 1.959 & & \\
\hline & 727.742 & 0.0 & 1.973 & & & & & & & 44.97 & 1.989 & & \\
\hline & 1060.770 & & & & & & & & & 0.0 & 2.155 & & \\
\hline \multirow[t]{8}{*}{$10^{2}$} & 0 & 91.31 & 2.166 & 89.74 & 2.152 & 46.81 & 2.485 & 37.79 & 2.452 & 155.29 & 2.001 & 64.33 & 2.254 \\
\hline & 100 & 80.98 & 2.154 & 78.74 & 2.137 & 36.19 & 2.357 & 23.33 & 2.284 & 14 & 2.009 & 53.25 & 2.228 \\
\hline & 223.195 & 68.02 & 2.145 & 64.69 & 2.124 & 21.75 & 2.261 & 0.0 & 2.295 & 128.83 & 2.023 & 39.35 & 2.202 \\
\hline & 394.100 & 49.59 & 2.140 & 44.12 & 2.121 & 0.0 & 2.200 & & & 107.93 & 2.049 & 19.62 & 2.179 \\
\hline & 559.742 & 31.22 & 2.147 & 22.68 & 2.148 & & & & & 87.05 & 2.084 & 0.0 & 2.171 \\
\hline & 720.941 & 12.82 & 2.165 & 0.0 & 2.221 & & & & & 66.05 & 2.131 & & \\
\hline & 830.388 & 0.0 & 2.185 & & & & & & & 51.33 & 2.172 & & \\
\hline & 1185.020 & & & & & & & & & 0.0 & 2.379 & & \\
\hline \multirow[t]{8}{*}{$10^{3}$} & 0 & 163.11 & 2.971 & 160.43 & 2.953 & 66.36 & 3.605 & 54.48 & 3.541 & 329.35 & 2.718 & 107.81 & 3.086 \\
\hline & 100 & 154.34 & 2.963 & 151.27 & 2.943 & 59.08 & 3.469 & 44.63 & 3.348 & 316.99 & 2.726 & 98.55 & 3.068 \\
\hline & 392.315 & 127.60 & 2.950 & 122.89 & 2.923 & 33.68 & 3.189 & 0.0 & 3.358 & 279.22 & 2.758 & 70.41 & 3.034 \\
\hline & 711.215 & 96.29 & 2.959 & 88.32 & 2.935 & 0.0 & 3.102 & & & 234.71 & 2.814 & 37.76 & 3.033 \\
\hline & 1054.550 & 59.65 & 3.003 & 43.28 & 3.035 & & & & & 181.78 & 2.909 & 0.0 & 3.076 \\
\hline & 1287.194 & 32.69 & 3.054 & 0.0 & 3.274 & & & & & 141.94 & 3.001 & & \\
\hline & 1546.810 & 0.0 & 3.136 & & & & & & & 92.15 & 3.138 & & \\
\hline & 1939.690 & & & & & & & & & 0.0 & 3.423 & & \\
\hline
\end{tabular}

$\mathrm{Ma}_{\mathrm{c} 4}<\mathrm{Ma}_{\mathrm{c} 3}<\mathrm{Ma}_{\mathrm{c} 6}<\mathrm{Ma}_{\mathrm{c} 2}<\mathrm{Ma}_{\mathrm{c} 1}<\mathrm{Ma}_{\mathrm{c} 5}$

for both ferro and non-ferrofluids. That is, the system is most unstable (i. e., augments convection) in the case of step function type of basic temperature gradient because the jump in temperature occurs nearer the less restrictive free surface, whereas the inverted parabolic type of basic temperature gradient makes the system more stable. Thus, it is possible to control Marangoni ferroconvection effectively by the choice of different forms of basic temperature gradients including the effect of Coriolis force due to rotation. From the tables it is also noted that the critical wavenumber decreases with the increase in the value of $N$ but the decreasing trend with $N$ is not so significant, but an increase in the value of Ta significantly increases the critical wavenumber. Hence the effect of an increase in Ta and decrease in $N$ is to reduce the size of the convection cells.

The variation of critical Marangoni numbers $\mathrm{Ma}_{\mathrm{c} 2}, \mathrm{Ma}_{\mathrm{c} 3}$ and $\mathrm{Ma}_{\mathrm{c} 4}$ as a function of thermal depth $\varepsilon$ showed that all of them decrease at first to a minimum value, and then increase steadily with increasing $\varepsilon$. The critical thermal depth $\varepsilon_{\mathrm{c}}$ obtained numerically for piecewise linear and step function temperature profiles are listed in Table 5 for different values of Ta and $N$. It can be seen that an increase in Ta is to increase $\varepsilon_{c}$, while an increase in $N$ is to decrease the same. 
Table 5. Values of $\varepsilon_{\mathrm{c}}$ for different values of Ta and $N$ when $M_{3}=1$

\begin{tabular}{lclll}
\hline Nature of temperature Profiles & Ta & $\begin{array}{l}N=0 \\
\varepsilon_{\mathrm{c}}\end{array}$ & $\begin{array}{l}N=100 \\
\varepsilon_{\mathrm{c}}\end{array}$ & $\begin{array}{l}N=150 \\
\varepsilon_{\mathrm{c}}\end{array}$ \\
\hline Piecewise linear profile for heating from below & 1 & 0.959 & 0.948 & 0.941 \\
& 10 & 0.960 & 0.949 & 0.943 \\
Piecewise linear profile for cooling from above & $10^{2}$ & 0.964 & 0.955 & 0.951 \\
& $10^{3}$ & 0.980 & 0.977 & 0.976 \\
Step function profile & 10 & 0.678 & 0.617 & 0.592 \\
& $10^{2}$ & 0.680 & 0.620 & 0.595 \\
& $10^{3}$ & 0.701 & 0.656 & 0.622 \\
& 1 & 0.812 & 0.760 & 0.745 \\
& 10 & 0.814 & 0.749 & 0.724 \\
& $10^{2}$ & 0.827 & 0.770 & 0.726 \\
\hline
\end{tabular}

\section{Acknowledgements}

This work was supported by the UGC-Centre for Advanced Studies in Fluid Mechanics. One of the authors (C.E.N.) would like to thank the Principal and Management of Dr. Ambedkar Institute of Technology, Bangalore, for their encouragement.

\section{References}

[1] Rosensweig, R. E.: Ferrohydrodynamics. Cambridge: Cambridge University Press 1985.

[2] Berkovsky, B. M., Medvedev, V. F., Krakov, M. S.: Magnetic fluids. Engineering applications. Oxford University Press 1993.

[3] Bashtovoy, V. G., Berkovsky, B. N., Vislovich, A. N.: Introduction to thermomechanics of magnetic fluids. Washington, D. C.: Hemisphere Co. 1988.

[4] Finlayson, B. A.: Convective instability of ferromagnetic fluids. J. Fluid Mech. 40, 753-767 (1970).

[5] Lalas, D. P., Carmi, S.: Thermoconvective stability of ferrofluids. Phys. Fluids 4, 436-438 (1971).

[6] Gotoh, K., Yamada, M.: Thermal convection in a horizontal layer of magnetic fluids. J. Phys. Soc. Japan 51, 3042-3048 (1982).

[7] Schwab, L., Hilderbrandt, U., Stierstadt, K.: Magnetic Benard-convection. J. Magn. Magn. Mater. 65, 315-319 (1987).

[8] Stiles, P. J., Kagan, M.: Thermoconvective instability of a ferrofluid in a strong magnetic field. J. Colloid Interface Sci. 134, 435-448 (1990).

[9] Kaloni, P. N., Lou, J. X.: Convective instability of magnetic fluids. Phys. Rev. E 70, 026313026324 (2004).

[10] Das Gupta, M., Gupta, A. S.: Convective instability of a layer of a ferromagnetic fluid rotating about a vertical axis. Int. J. Engng Sci. 17, 271-277 (1979).

[11] Rudraiah, N., Sekhar, G. N.: Convection in magnetic fluids with internal heat generation. ASME. J. Heat Transfer 113, 122-127 (1991).

[12] Venkatasubramanian, S., Kaloni, P. N.: Effects of rotation on the thermoconvective instability of a horizontal layer of ferrofluids. Int. J. Engng Sci. 32, 237-256 (1994).

[13] Sekar, R., Vaidyanathan, G., Ramanathan, A.: The ferroconvection in fluids saturating a rotating densely packed porous medium. Int. J. Engng Sci. 31, 241-250 (1993).

[14] Vaidyanathan, G., Sekar, R., Vasnthakumari, R., Ramanathan, A.: The effect of magnetic field dependent viscosity on ferrconvection in a rotating sparsely distributed porous medium. J. Magn. Magn. Mater. 250, 65-76 (2002).

[15] Kaloni, P. N., Lou, J. X.: Weakly nonlinear instability of ferromagnetic fluids rotating about a vertical axis. J. Magn. Magn. Mater. 284, 54-68 (2004). 
[16] Pearson, J. R. A.: On convection cells induced by surface tension. J. Fluid Mech. 4, 489-501 (1958).

[17] Qin, Y., Kaloni, P. N.: Nonlinear stability problem of a ferromagnetic fluid with surface tension effect. Eur. J. Mech. B/Fluids 13, 305-321 (1994).

[18] Shivakumara, I. S., Rudraiah, N., Nanjundappa, C. E.: Effect of nonuniform basic temperature gradient on Rayleigh-Benard-Marangoni convection in ferrofluids. J. Magn. Magn. Mater. 248, 379-395 (2002).

[19] Lebon, G., Cloot, A.: Effects of nonuniform temperature gradients on Benard-Marangoni instability. J. Non-Equilib. Thermodyn. 6, 15-30 (1981).

[20] Vidal, A., Acrivos, A.: The influence of Coriolis force on surface tension driven convection. J. Fluid Mech. 26, 807-818 (1966).

Authors' addresses: I. S. Shivakumara, UGC-Centre for Advanced Studies in Fluid Mechanics, Department of Mathematics, Bangalore University, Central College Campus, Bangalore-560 001, India (E-mail: isshivakumara@hotmail.com); C. E. Nanjundappa, Department of Mathematics, Dr. Ambedkar Institute of Technology, Bangalore-560 056, India 